\title{
EFEITOS DA EXPLORAÇÃO CONVENCIONAL E DE IMPACTO REDUZIDO EM UMA FLORESTA ESTACIONAL SEMIDECIDUAL NA PROVÍNCIA DE MISIONES, NORDESTE DA ARGENTINA
}

\author{
Nardia Maria Luján Bulfe ${ }^{1}$, Franklin Galvão², Afonso Figueiredo Filho ${ }^{3}$, Patrício Mac Donagh ${ }^{4}$ \\ ${ }^{1}$ Eng ${ }^{\text {a }}$ Florestal, M.Sc., Depto. Forestal, INTA - nbulfe@ montecarlo.inta.gov.ar \\ ${ }^{2}$ Eng. Florestal, Dr., Depto. de Ciências Florestais, UFPR - fgalvao@ufpr.br \\ ${ }^{3}$ Eng. Florestal, Dr., Depto. de Engenharia Florestal, UNICENTRO - afonso.figueiredo@pq.cnpq.br \\ ${ }^{4}$ Eng. Florestal, M.Sc., Área de Producción y Economia, UNaM - mdonagh@ facfor.unam.edu.ar \\ Recebido para publicação: 20/06/2008 - Aceito para publicação: 27/10/2008
}

\begin{abstract}
Resumo
O presente estudo foi desenvolvido na Reserva Florestal de Uso Múltiplo Guarani, na província de Misiones, Argentina. Foram instaladas 15 parcelas permanentes, de quatro hectares cada, com uma área efetiva de medição de um hectare, distribuídas em três tratamentos: Exploração Convencional (EC), Exploração de Impacto Reduzido (EIR) e Testemunha. Foram considerados todos os indivíduos arbóreos com diâmetro à altura do peito (DAP) $\geq 10 \mathrm{~cm}$ um ano antes da exploração, em 1998, e oito anos depois, em 2006. A exploração criou uma superfície total de clareiras de $8.018,7 \mathrm{~m}^{2}$, representando $6,7 \%$ da área total amostrada, 65\% gerados pela EC. A área basal danificada foi superior na EC (70\% do total). A EIR concentrou as clareiras em classes de tamanhos inferiores, com áreas médias menores. O número de indivíduos danificados foi de 13,6 ind.ha ${ }^{-1}$, com uma percentagem superior de danos ocasionada pela EC. O abate do indivíduo foi a principal causa do dano, e a parte da árvore mais danificada, a copa. Determinou-se uma relação positiva e significativa entre a área basal explorada e a área basal danificada $\left(R^{2}=0,49\right)$. $O$ planejamento da exploração é o fator principal na redução dos danos aos indivíduos remanescentes, sendo o abate direcionando a queda da árvore uma das técnicas que minimiza os danos.
\end{abstract}

Palavras-chave: Clareiras; danos por exploração; exploração de impacto reduzido; Floresta Estacional Semidecidual.

\begin{abstract}
Effect of conventional and reduced impact logging in the semi deciduous forest of Misiones, northeast of Argentine. The present study was developed in the Multiple Use Guarani Forest Reserve, in Misiones, Argentine. There, 15 permanent plots of four hectare each one were installed. Each plot had an effective area of one hectare measured and it was distributed in three treatments: Conventional Logging, Reduced Impact Logging and control. At the year of logging (1999) and eight years later (2006), all the trees larger than $10 \mathrm{~cm}$ of diameter at breast height (DBH) were measured. The logging created a total surface of gaps of $8,018.7 \mathrm{~m}^{2}$, which represented $6.7 \%$ of the total sampled area, where $65 \%$ were generated by the Conventional Logging. The basal area damaged was higher in the Conventional Logging treatment (70\% of the total). The Reduced Impact Logging concentrates the gaps on classes of lower sizes and in smaller average areas. The number of damaged individuals was 13.6 stems.ha ${ }^{-1}$, with a higher percentage of damages caused by the Conventional Logging. The cut of individuals was the main cause of damage and the crown was the most damaged part of the tree. A positive and significant relation was determined between the logging basal area and the damaged basal area $\left(\mathrm{R}^{2}=0.49\right)$. The planning of the logging showed to be the main factor in the reduction of the hold over stem damages. Beyond that, the slaughter directing the tree fall showed to be one of the techniques that reduce damages.

Keywords: Treefall gaps; logging damage; reduced impact logging; neotropical forest.
\end{abstract}

\section{INTRODUÇÃO}

A maioria das operações de exploração nas florestas tropicais e subtropicais onde a legislação é permissiva não incorpora métodos para minimizar os danos da exploração (WEBB, 1997), os quais levam as florestas a um estado de alto grau de degradação. Por outro lado, pesquisas na Indonésia (BERTAULT; 
SIST, 1995; BERTAULT; SIST, 1997; SIST et al., 1998; SIST; NGUYEN-THÉ, 2002), na Costa Rica (WEBB, 1997) e no Brasil (JOHNS et al., 1996; PEREIRA et al., 2002; SIST; FERREIRA, 2007) revelam que há possibilidades de reduzir significativamente os danos aos remanescentes durante as operações de exploração. Assim, uma intervenção conservacionista da floresta incrementa as possibilidades do uso sustentável dos recursos (WEBB, 1997).

Sempre que ocorre a formação de uma clareira, além de provocar danos à vegetação remanescente mais próxima, mudanças no comportamento dos indivíduos são observadas (SIST; NGUYEN-THÉ, 2002; VIDAL et al., 2002; SIST; FERREIRA, 2007). Quanto à intervenção na floresta, uma redução drástica na área basal causaria uma mudança importante na dinâmica dos indivíduos e na composição florística, razão pela qual se estima que a redução da área basal não deve exceder a $15 \%$ (SIST; NGUYEN-THÉ, 2002).

Essas mudanças estariam relacionadas principalmente ao grau de distúrbio, sendo este decorrente da intensidade de exploração (SIST; NGUYEN-THÉ, 2002) e das técnicas utilizadas (MAC DONAGH et al., 2005). Assim, um maior grau de distúrbio provocaria um crescimento maior nos remanescentes (SIST; NGUYEN-THÉ, 2002).

Nesse marco conceitual, Sist; Ferreira (2007) mencionam, restringindo-se à produção, que para uma floresta na Amazônia com uma intensidade de exploração de três árvores por hectare e com um crescimento médio em diâmetro de $4 \mathrm{~mm}$ por ano, um ciclo de 40 anos poderia ser suficiente para uma nova intervenção. Os mesmos autores mencionam que, quando considerado um ciclo de corte de 30 anos, a intensidade da segunda intervenção deveria ser de 2 ou 3 árvores por hectare, sempre que a floresta atingir crescimentos de 3,5 e $5 \mathrm{~mm}$ por ano, respectivamente. Mas, sabe-se que atingir esse crescimento nos remanescentes, sem a implementação de tratamentos silviculturais pós-exploração, seria difícil (SIST; FERREIRA, 2007).

Vários são os fatores que influenciam no crescimento dos indivíduos arbóreos. Entre eles, Sist; Nguyen-Thé (2002) mencionam que o incremento diamétrico das espécies depende do tamanho do indivíduo, em que a distribuição do incremento diamétrico médio nas classes diamétricas vai incrementando com o DAP. Dessa forma, indivíduos pequenos (10-19 $\mathrm{cm}$ de DAP) apresentam os menores incrementos, enquanto que aqueles com DAP $\geq 50 \mathrm{~cm}$ são os que têm maiores incrementos.

Por outro lado, num estudo realizado na Amazônia oriental do Brasil, durante um período de três anos, Vidal et al. (2002) determinaram que a perturbação causada por uma exploração sem manejo controlado provocou danos e alterações biofísicas, resultando, assim, numa diminuição no crescimento das árvores remanescentes. Os autores observaram que o crescimento médio em diâmetro dos indivíduos foi de $0,63,0,37$ e $0,33 \mathrm{~cm}^{-a n o}{ }^{-1}$ para áreas com e sem manejo controlado e áreas-testemunha, respectivamente. Nomearam como áreas sem manejo controlado aquelas onde não foi aplicado nenhum tratamento antes, durante e após a exploração; e como áreas manejadas aquelas onde foram executadas tarefas de planejamento.

Conforme Johns et al. (1996) e Webb (1997), um conjunto de atividades pré-exploração e técnicas de manejo podem reduzir os danos em todas as fases de exploração. Entre as de maior importância, esses autores citam o corte de lianas, o abate dirigido, o tipo de maquinaria utilizada, o planejamento e traçado de caminhos de extração e a construção de área de estoque. Outros autores mencionam que a abertura e o dano residual são minimizados quando as clareiras se concentram numa área (JACKSON et al., 2002) e citam como técnica fundamental para minimizar os danos aos remanescentes, que passam a ser um dos componentes críticos da exploração florestal de impacto reduzido, o abate dirigido das árvores exploradas (PEREIRA et al., 2002).

Conforme Bertault; Sist (1997), há uma correlação positiva e significativa entre a proporção de árvores danificadas e a área basal extraída. Concordando com esses autores, Sist; Nguyen-thé (2002) mencionam que a taxa de mortalidade em áreas exploradas é influenciada por uma maior mortalidade de árvores danificadas, principalmente daquelas com danos severos. Assim, os autores comprovaram que áreas com uma intensidade de exploração baixa e controlada, e dessa forma com menores danos, tiveram taxas de mortalidade similares às observadas nas áreas-testemunhas sem intervenção (1,5\%). Com isso, fica estabelecido que uma redução nos danos diminui a mortalidade pós-exploração (BERTAULT; SIST, 1997; SIST et al., 1998; SIST; NGUYEN-THÉ, 2002).

Segundo Sist; Nguyen-Thé (2002), o recrutamento aumenta na medida em que a intensidade de exploração aumenta. Em áreas-testemunha, observaram uma média de 8 árvores por hectare, enquanto 
que em áreas exploradas foram registradas de 14 até 33 árvores por hectare (>80\% e $<70 \%$ da área basal extraída, respectivamente).

Há uma relação positiva e significativa entre as árvores danificadas e a intensidade de exploração ( $r=0,62$ para BERTAULT; SIST, 1997; e $r=0,59$ para SIST et al., 1998). Dessa forma, a intensidade de exploração é uma característica importante quanto aos danos causados pela exploração, independentemente da técnica utilizada (SIST et al., 1998).

Quanto à resposta dos indivíduos danificados, eles apresentam um comportamento diferenciado do comportamento daqueles sem danos. Vidal et al. (2002) mencionam que o crescimento diamétrico médio de árvores sem danos poderia atingir valores de $0,72 \mathrm{~cm}^{-a n o}{ }^{-1}$ e de 0,51 e $0,33 \mathrm{~cm} \cdot$ ano $^{-1}$, quando apresentam danos leves e severos, respectivamente, sendo essas diferenças em crescimento estatisticamente significativas.

Assim, fica demonstrada a importância da ligação entre a evolução da floresta e as técnicas utilizadas antes, durante e após a exploração. Nesse contexto, os danos são a principal influência da evolução da massa após a exploração, principalmente para definir o ciclo de exploração e a utilização sustentável dos recursos florestais.

Estudos desenvolvidos no Brasil, Suriname e Malásia promovem a evidência de que o planejamento e o controle na exploração podem reduzir efetivamente os danos aos remanescentes em vários tipos de florestas tropicais (SIST et al., 1998; JOHNS et al., 1996; BERTAULT; SIST, 1997; VIDAL et al., 2002).

Sem planejamento na exploração, esperam-se obter até 48\% de árvores danificadas (SIST et al., 1998; VIDAL et al., 2002), podendo ser reduzidos para 30\% quando são aplicadas técnicas de impacto reduzido (SIST et al., 1998). Entre os danos mais comuns provocados durante a exploração, estão os danos à copa, seguidos pelos danos à casca e ao fuste (BERTAULT; SIST, 1997).

As diferentes operações feitas durante a exploração provocam danos de forma não proporcional aos remanescentes. Dessa forma, e conforme Johns et al. (1996) e Bertault; Sist (1997), as operações de exploração que mais danos provocam à floresta são o abate e a extração do fuste. Segundo Sist et al. (1998) e Bertault; Sist (1997), a operação de abate ocasiona principalmente danos à copa (48\%), enquanto que a operação de extração foi a principal causa de mortalidade (60\%).

Quando se faz a análise da distribuição dos danos nas diferentes classes diamétricas, Bertault; Sist (1997) mencionam que eles não foram distribuídos de acordo com a abundância relativa em cada uma delas, apresentando uma alta frequência de danos nas classes intermediárias (30-50 cm DAP). Por outro lado, e com resultados antagônicos aos anteriores, Sist; Ferreira (2007) informam que os danos se distribuíram em cada classe diamétrica de acordo com a abundância arbórea relativa, sugerindo, assim, que todas as classes diamétricas podem ser afetadas na mesma amplitude.

A cobertura do dossel de uma floresta sem intervenção poderia atingir valores de cobertura de 91,4\%, enquanto que uma floresta explorada, dependendo da intensidade de exploração, atingiria somente 73,4\% (JOHNS et al., 1996).

Quanto à abertura provocada no dossel pela exploração, Johns et al. (1996) comprovaram que a queda de uma árvore acarreta aberturas médias menores em área com planejamento quando comparada com áreas sem (166 e $355 \mathrm{~m}^{2}$, respectivamente), atingindo uma superfície total de clareiras de 845 e 1739 $\mathrm{m}^{2}$ cada, correspondendo esses valores a uma perda de dossel estimada de $10 \%$ e $19 \%$, respectivamente. De forma análoga, Webb (1997) observou uma relação linear negativa entre a cobertura de dossel pósexploração e a intensidade de exploração $\left(\mathrm{R}^{2}=0,82\right)$.

Conforme Johns et al. (1996), espera-se que a reconstituição do dossel de clareiras grandes seja feita por uma maior proporção de pequenas árvores e plântulas quando comparadas com clareiras pequenas. Como resultado, o fechamento da clareira por espécies que pertencem à fase madura poderia levar mais tempo numa clareira grande que numa pequena. Mas o nível de recuperação, naturalmente, decresce quando se incrementa a taxa de danos por exploração (SIST; FERREIRA, 2007).

Dessa forma, resulta essencial o monitoramento e tratamentos silviculturais das árvores de futuro, classe de DAP média, as quais serão exploradas, uma vez que permitiriam melhorar as técnicas do impacto reduzido, ajudando na redução dos danos, na sobrevivência e num maior crescimento dos indivíduos remanescentes (BERTAULT; SIST, 1997). 
Este trabalho foi concebido com o propósito de avaliar os efeitos da exploração convencional e de impacto reduzido aplicados em uma Floresta Estacional Semidecidual quanto à cobertura do dossel, à florística e aos danos causados nas árvores remanescentes.

\section{MATERIAL E MÉTODOS}

\section{Área de estudo}

O presente estudo foi desenvolvido na Reserva Florestal de Uso Múltiplo Guarani, que está inserida na Reserva de Biosfera Yabotí, na província de Misiones, Argentina. A área pertence ao departamento Guaraní, município El Soberbio, tem 5.343 ha, e sua posição geográfica é $26^{\circ} 57^{\prime} \mathrm{S}$ e $54^{\circ} 15^{\prime}$ W, tendo como limite ao norte o arroio Paraíso e ao sul o arroio El Soberbio.

De forma geral, a cobertura da área compreende cerca de $90 \%$ de floresta em diferentes estados de conservação; $2 \%$ de infra-estrutura, incluindo a rodovia que atravessa a unidade; e $8 \%$ ocupados por uma comunidade indígena (GAUTO, 1997).

A paisagem dominante na região é serrana, com relevo moderado a escarpado. Na reserva, $63 \%$ da superfície têm declividade inferior a $15 \%, 32 \%$ enquadram-se entre $15 \%$ e $45 \%$, e $5 \%$ superam $45 \%$ de declividade. A altitude varia entre $267 \mathrm{~m}$ s.n.m. e 574 m. s.n.m., sendo que a metade da área acha-se compreendida entre 400 e 500 m s.n.m. $37 \%$ correspondem à altitude entre 300 e 400 m s.n.m., sendo o complemento de $14 \%$ distribuídos entre as porções mais altas e mais baixas (PALAVECINO; MAIOCCO, 1995).

\section{Clima da região}

O clima da região corresponde, segundo Köppen, ao tipo $\mathrm{C} f a$, sendo subtropical mesotérmico, sem estação seca. A precipitação média anual é de $1800 \mathrm{~mm}$, distribuídos proporcionalmente em todos os meses do ano. A temperatura média anual do ar é de $21^{\circ} \mathrm{C}$, apresentando uma média de $25^{\circ} \mathrm{C}$ no mês mais quente (janeiro), com máximas absolutas de $39^{\circ} \mathrm{C}$, e de $14{ }^{\circ} \mathrm{C}$ no mês mais frio (julho), com mínima absoluta de $-6^{\circ} \mathrm{C}$ (EIBL et al., 1995).

\section{Solos}

Segundo Ligier et al. (1990), o conjunto dos solos com maior predominância em Misiones, os quais representam 32,7\% da superfície total da província, são os Argissolos, Luvissolos e Nitossolos. Quanto à sua aptidão, esses solos, oriundos de rochas ígneas extrusivas, são considerados como os melhores para a atividade agrícola e florestal. São comuns também os Chernossolos, Neossolos e Cambissolos. Eles são dominantes em regiões com relevos dissecados.

$\mathrm{Na}$ área de estudo, os solos enquadram-se em seis ordens: Cambissolos (31\%), Neossolos (20\%), Chernossolos (18\%), Luvissolos (13,4\%), Argissolos (8\%) e Nitossolos (6,6\%). Os restantes 3\% pertencem às áreas com presença de afloramentos de rocha. A reserva acha-se coberta por solos menos desenvolvidos, quanto à sua gênese, sendo os Cambissolos os mais abundantes, seguidos pelos Neossolos e Chernossolos (PAHR et al., 1997).

\section{Vegetação}

Segundo Cabrera (1951), a província de Misiones está inserida por completo nos limites da Província Subtropical Oriental, denominação que abarca parte do sudoeste do Brasil, leste do Paraguai e nordeste da Argentina.

Com base em Martínez-Crovetto (1963), a área de estudo enquadra-se no Setor Missioneiro da Província Subtropical Oriental, onde a vegetação é florestal e comum aos estados do Rio Grande do Sul, Santa Catarina e Paraná, ao leste do Paraguai e à porção ocidental da província de Misiones. A Província Subtropical Oriental é complementada pelo Setor Planaltense, que compreende os chamados planaltos austro-brasileiros e a porção norte da província de Misiones, e pelo Setor Litoral Atlântico, que se estende seguindo a Serra do Mar até o sul do estado do Rio Grande do Sul.

De acordo com a classificação feita por Cabrera; Willink (1980), a área está localizada na região fitogeográfica denominada "Província Paranaense", distrito das selvas, e, conforme a terminologia sugerida por Veloso; Góes-Filho (1982), a região de estudo é coberta de Floresta Estacional Semidecidual, representada por diferentes estratos. 
Mac Donagh et al. (dados não publicados) determinaram, para a área na qual foi desenvolvida essa pesquisa, a presença de 102 espécies arbóreas e uma média de 46 espécies por hectare, com as mais representativas sendo as das famílias Papilionaceae (14\%), Lauraceae (13\%), Rutaceae (9\%), Meliaceae (8\%), Euphorbiaceae, Sapindaceae (7\%) e Sapotaceae (6\%). Tressens et al. (1997) encontraram, numa área próxima, 114 espécies arbóreas, distribuídas em 92 gêneros, sendo representadas por 42 famílias.

\section{Descrição do ensaio}

Foram instaladas 15 parcelas permanentes, quadradas, de quatro hectares $(200 \times 200 \mathrm{~m})$ cada, o que totaliza uma área experimental de 60 hectares. Cada parcela tem uma área efetiva de medição de um hectare, com uma área de bordadura de $50 \mathrm{~m}$ (Figura 1).

As parcelas foram submetidas a metodologias de exploração diferenciadas, o que resultou nos seguintes tratamentos:

1) Exploração de Impacto Reduzido (7 parcelas) - Esse tratamento é baseado no planejamento e controle da implementação das operações de exploração, com a finalidade de minimizar o impacto sobre a floresta remanescente e o solo. Em função disso, antecedendo à exploração, foram efetivadas atividades de planejamento das estradas e trilhas de arrastes e dos pátios de estocagem. Entre as principais atividades de exploração, a de maior importância foi o abate direcional das árvores exploradas, com o fim de evitar danos aos indivíduos remanescentes.

2) Exploração Convencional (5 parcelas) - Nesse tratamento, o operário (empreiteiro) selecionou livremente os indivíduos a serem abatidos, o tráfego de máquinas e a localização dos pátios de estocagem no momento da exploração.

3) Testemunha (3 parcelas) - Nesse tratamento, nenhuma árvore foi abatida e não houve trânsito de máquinas nas parcelas.

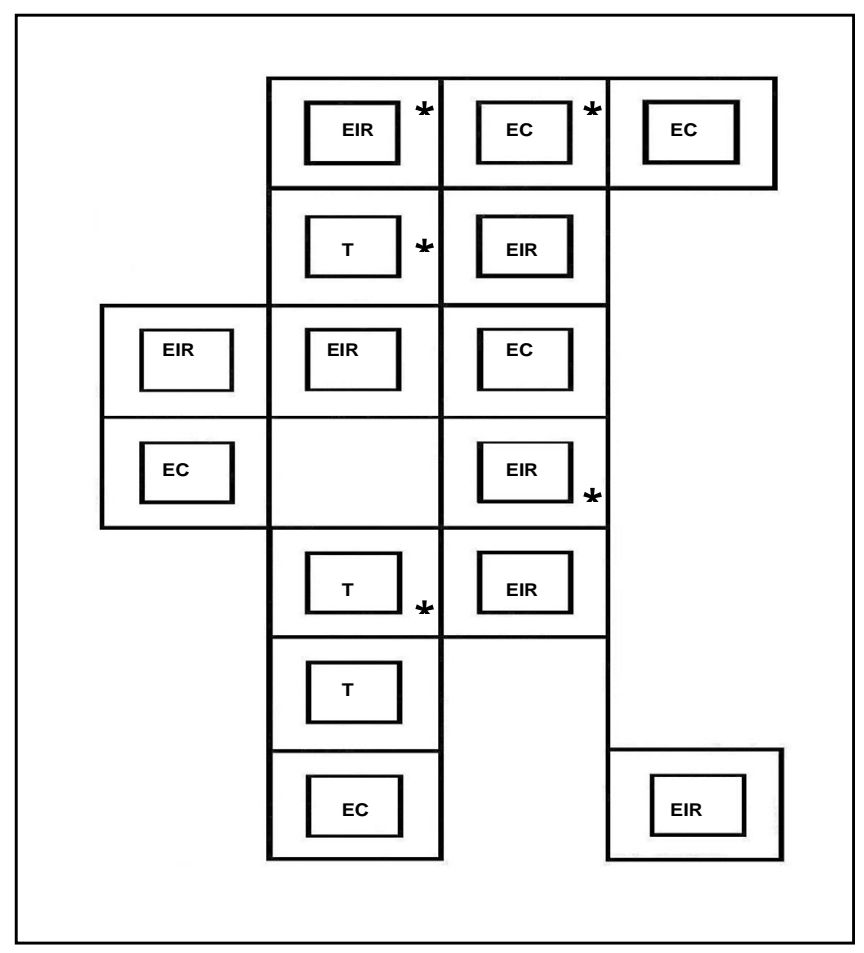

Figura 1. Desenho experimental da área de estudo, na Reserva de Uso Múltiplo Guarani. EIR: Exploração de Impacto Reduzido; EC: Exploração Convencional; T: Testemunha. *: Parcelas onde foram feitas fotografias hemisféricas.

Figure 1. Experimental design of the study area, in the Guarani Forest Reserve. EIR: Reduced Impact Logging; EC: Conventional Logging; T: Control Plot. *: Plots where hemispherical photographs were taken. 
No estudo, consideraram-se unicamente os indivíduos estabelecidos dentro da área efetiva de medição de um hectare.

Durante os meses de junho e julho de 1999, foi realizada a exploração, quando foram abatidos, nos diferentes tratamentos, um total de 45 indivíduos, pertencentes a 11 espécies diferentes e a 7 famílias (Tabela 1).

As espécies foram colhidas levando-se em consideração o diâmetro mínimo de corte (DMC) determinado pelo Ministério de Ecologia da província de Misiones, segundo o qual cada espécie estabelecida na lista das comerciais deve apresentar um diâmetro mínimo a partir do qual pode ser explorada, variável de espécie para espécie.

\section{Obtenção de dados e estabelecimento de critérios}

Antecedendo a exploração, em 1998 foram mapeados e medidos todos os exemplares arbóreos com DAP $\geq 10 \mathrm{~cm}$, sendo remedidos em 2006. Todos os indivíduos foram identificados em nível de espécie, sendo cada exemplar localizado através de coordenadas e marcado com uma plaqueta de alumínio.

Após a exploração, em 2000, foram registrados os danos nos indivíduos remanescentes, utilizando-se a metodologia estabelecida por Bertault; Sist (1997), a qual considera a origem do dano (queda ou arraste) e a parte da árvore afetada (copa ou fuste) em percentagem.

Para verificar a influência dos sistemas de exploração, definiu-se "clareira" conforme Brokaw (1982), que considera como sendo aquela área do dossel completamente aberta a partir de dois metros sobre o nível do solo. No ano de 2005, foram definidos os limites das clareiras ao redor do toco remanescente da árvore abatida, utilizando-se metodologia desenvolvida por Runkle (1981), que consiste em determinar o comprimento da clareira, coincidente com a orientação da queda da árvore, e a largura, a qual é medida no ponto médio transversal ao comprimento. Assim foi estimada a sua área mediante o uso da fórmula da elipse: Área $\left(m^{2}\right)=(\pi \cdot$ comprimento $\cdot$ largura $) / 4$.

Para a análise de cobertura do dossel, no mês de outubro de 2007 foram tomadas fotografias hemisféricas em cinco parcelas, representando Exploração de Impacto Reduzido, Exploração Convencional e Testemunha. A câmera utilizada foi uma câmera digital marca Nikon Coolpix950, montada sobre um equipamento autonivelador (Delta-T Devices Ltd., Cambridge, UK), com uma lente Nikkor $8 \mathrm{~mm}$. As fotos foram tiradas em 6 pontos em cada parcela, com uma distância de 20 metros entre pontos, em dois transectos de 100 metros sorteados ao acaso, e a duas alturas, 0,7 e 1,5 metros. A distância entre fotos de 20 metros foi adotada para dar a segurança da independência entre fotos contíguas (CLARK et al., 1996, citado em CAMPANELLO, 2004). As fotografias foram analisadas com o programa Hemiview (Delta-T Devices Ltd., Cambridge, UK). Foi determinada a percentagem de cobertura de dossel arbóreo, para o qual foi feita a análise ANOVA entre tratamentos.

\section{Processamento de dados}

A fim de descrever os tratamentos, determinou-se para cada um deles seus parâmetros estruturais, a superfície total de clareiras $\left(\mathrm{m}^{2}\right)$, a área basal explorada e a área basal danificada $\left(\mathrm{m}^{2}\right)$. Determinou-se a frequência por classe de tamanho de clareiras para cada tratamento, além das análises de regressão entre a área da clareira e o DAP $(\mathrm{cm})$ da árvore abatida, e entre o nível do dano e a área da clareira.

\section{RESULTADOS E DISCUSSÃO}

\section{Florística}

No total do ensaio, foram registradas 85 espécies diferentes, distribuídas em 34 famílias. Em cada um dos tratamentos foram identificadas 73 espécies na Exploração Convencional, 65 na Exploração de Impacto Reduzido e 68 na testemunha. Do total das espécies encontradas em todo o ensaio, 58\% delas

${ }^{1}$ CLARK, D. B.; CLARK, D. A.; RICH, D. A.; WEISS, S.; OBERBAUER, S. F. Landscape scale avaluation of understory light and canopy structure: methods and application in a neotropical lowland rain forest. Canadian. Journal of Forest Research 26: $747-$ 757, 1996. 
foram comuns aos três tratamentos, com destaque, pela maior densidade, para Nectandra megapotamica, Lonchocarpus leucanthus, Balfourodendron riedelianum e Trichillia catigua, tendo as duas primeiras maior densidade nas parcelas testemunhas. Gauto (1997), num local de estudo muito próximo ao desta pesquisa, identificou um total de 72 espécies arbóreas em 4 ha sob exploração, onde as espécies com maior número de indivíduos, excluindo Trichillia catigua, foram coincidentes com as aqui mencionadas, porém com densidades diferentes.

Quando realizada a exploração, foi abatido nos diferentes tratamentos um total de 45 indivíduos, pertencentes a 11 espécies diferentes, distribuídas em 7 famílias (Tabela 1). Toda árvore abatida foi despontada e transformada em toras de tamanho variável, as quais foram "amarradas" com cabo de aço e arrastadas até o pátio de armazenamento com auxílio de um skidder.

Tabela 1. Espécies exploradas nos diferentes sistemas de exploração e seus parâmetros estatísticos. Table1. Logged species in the different logging systems and their statistic parameters.

\begin{tabular}{llcc}
\hline Família & \multicolumn{1}{c}{ Espécie } & $\begin{array}{c}\text { Número de } \\
\text { indivíduos }\end{array}$ & DAP médio (cm) $\pm \boldsymbol{\sigma}$ \\
\hline Bignoniaceae & Tabebuia pulcherrima Sandwith & 1 & 89 \\
Fabaceae & Apuleia leiocarpa (Vogel) J.F. Macbr. & 9 & $87 \pm 18$ \\
& Parapiptadenia rigida (Benth.) Brenan & 13 & $73 \pm 13$ \\
& Myrocarpus frondosus Allemão & 1 & 95 \\
Lauraceae & Ocotea dyospirifolia (Meisn.) Mez & 2 & $60 \pm 0,5$ \\
& Ocotea puberula (Rich.) Nees & 4 & $71 \pm 0,9$ \\
Meliaceae & Cabralea canjerana (Vell.) Mart. & 1 & 81 \\
Poligonaceae & Cedrela fissilis Vell. & 2 & $62 \pm 19$ \\
Rutaceae & Bupretchia laxiflora Meisn. & 4 & $80 \pm 14$ \\
Tiliaceae & Luehea divaricata Mart. & 3 & $61 \pm 6$ \\
\hline Total & & 5 & $70 \pm 15$ \\
\hline$\sigma=$ desvio padrão. & & 45 & $75,4 \pm 15,5$
\end{tabular}

\section{Caracterização das clareiras}

Cada um dos indivíduos colhidos foi formador de uma clareira. Foi, assim, estudado um total de 45 clareiras artificiais.

As características gerais das clareiras estudadas e a área basal danificada são apresentadas na Tabela 2, sendo elas diferenciadas por tratamento de exploração.

Tabela 2. Caracterização das clareiras em cada modalidade de exploração.

Table 2. Gaps characteristics in each logging system.

\begin{tabular}{lcccccc}
\hline \multirow{2}{*}{ Tratamentos } & \multicolumn{2}{c}{ Área de clareiras $\left(\mathbf{m}^{\mathbf{2}}\right)^{*}$} & \multicolumn{3}{c}{ Área basal danificada $\left(\mathbf{m}^{\mathbf{2}}\right)^{*}$} \\
\cline { 2 - 7 } & Soma & Média & C.V. $(\%)$ & Soma & Média & C.V. $(\%)$ \\
\hline Exploração Convencional (EC) & $5.137,7$ & 197,6 & 49,4 & 3,48 & 0,13 & 97,8 \\
Exploração de Impacto Reduzido (EIR) & $2.881,0$ & 151,6 & 76,0 & 1,55 & 0,08 & 118,3 \\
\hline * Por área amostrada: EC = 7 ha; EIR = 5 ha; C.V.: coeficiente de variação. & & & &
\end{tabular}

A exploração criou uma superfície total de clareiras de $8.018,7 \mathrm{~m}^{2}$, o que representa $6,7 \%$ de uma área total de amostragem de $120.000 \mathrm{~m}^{2}$. Foi observada uma redução de $23,3 \%$ da superfície média gerada pelas clareiras no tratamento de Exploração de Impacto Reduzido, por hectare $\left(151,6 \mathrm{~m}^{2}\right.$ vs. 197,6 m²).

O tamanho médio de clareiras por modalidade de exploração é quase coincidente com os resultados obtidos em áreas com planejamento em uma floresta na Amazônia por Johns et al. (1996), que encontraram tamanhos de clareiras de $166 \mathrm{~m}^{2}$, diferindo quando a comparação é feita com áreas sem planejamento, em que encontraram valores médios muito superiores aos obtidos nesta pesquisa. Parte das causas que geraram tamanhos de clareiras discrepantes quando não é aplicada técnica para minimizar os danos, provavelmente se deve ao maior número de estratos e a coexistência de maior diversidade biológica na floresta Amazônica.

A área basal danificada foi superior no tratamento de Exploração Convencional, atingindo, após padronização, $62 \%$ do total dos danos ocasionados no ensaio (Tabela 2). Tendo em consideração que a 
queda direcionada das árvores exploradas é a técnica principal utilizada e o que diferencia os tratamentos de exploração, estaria sendo ela então a principal responsável pelas diferenças dos danos ocasionados entre os tratamentos, concordando, assim, com os autores Johns et al. (1996), Web (1997) e Pereira et al. (2002). Eles mencionam que uma das principais técnicas que promovem uma redução nos danos aos indivíduos remanescentes durante a exploração é o direcionamento de queda das árvores para proteger a regeneração de árvores de valor comercial e facilitar o arraste das toras. Dessa forma, os resultados aqui obtidos são semelhantes aos encontrados por Bertault; Sist (1995), Bertault; Sist (1997), Sist et al. (1998), Sist; Nguyen-Thé (2002), Pereira et al. (2002) e Sist; Ferreira (2007), que relatam a possibilidade de se reduzirem os danos quando a exploração é planejada.

Quanto ao tamanho das clareiras criadas durante a exploração, e concordando com o mencionado por Lawton; Putz (1988) e Ferreira Lima (2005), observou-se uma heterogeneidade na sua distribuição, com as classes de tamanho intermediário e acima de $230 \mathrm{~m}^{2}$ apresentando um maior número de clareiras. Na Figura 2 encontram-se esses valores especificados para cada tratamento. Nota-se que a Exploração Convencional apresenta um número maior de clareiras nas classes acima de $170 \mathrm{~m}^{2}$, tendo nessas classes, também, as maiores áreas médias de clareiras.
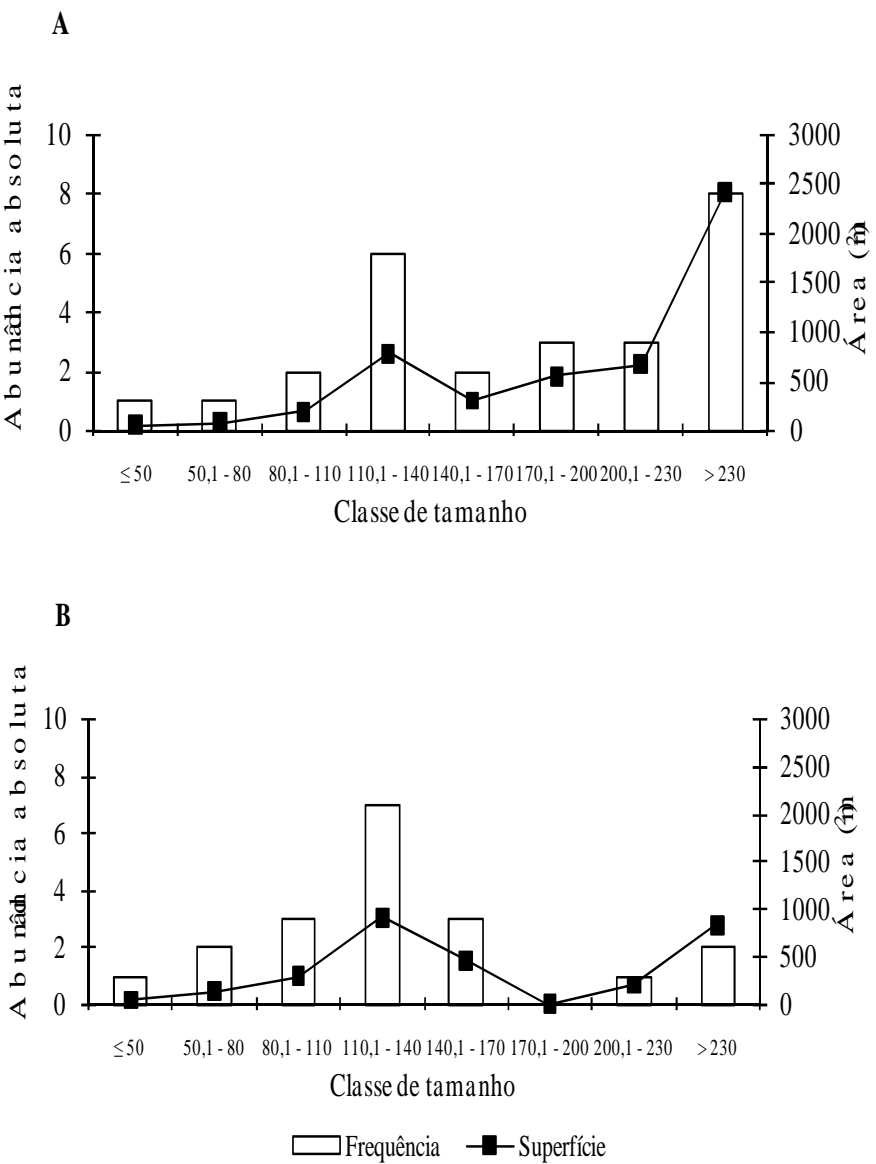

Figura 2. Frequência por classe de tamanho das clareiras resultantes dos tratamentos de exploração. A: Exploração Convencional; B: Exploração de Impacto Reduzido.

Figure 2. Frequency per gaps size classes in both logging treatments. A: Conventional Logging; B: Reduced Impact Logging.

Na Exploração de Impacto Reduzido, as clareiras estão concentradas nas classes de tamanhos abaixo de $140 \mathrm{~m}^{2}$ e com áreas médias também menores do que na Exploração Convencional. 
Essa heterogeneidade nas classes de tamanhos parece relacionar-se com dois fatores: o tamanho do indivíduo abatido, que resultaria em diferentes tamanhos de clareiras, e as técnicas de exploração utilizadas, que influenciariam na forma de distribuição das clareiras e nos tamanhos máximos que elas podem atingir.

Dessa forma, foi estabelecida a relação entre o tamanho do indivíduo abatido e a área da clareira criada por esse indivíduo. Observa-se que, neste estudo, o tamanho do indivíduo abatido não explica a variação do tamanho da clareira $\left(\mathrm{R}^{2}=0,0319 ; \mathrm{F}=1,418 ; \mathrm{N}=45\right)$. Quando analisados por tipo de exploração, novamente acontece a não ocorrência de uma relação entre ambas as variáveis, sendo os coeficientes de determinação para Exploração Convencional de 0,0705 $(\mathrm{F}=1,822 ; \mathrm{N}=26)$ e para Exploração de Impacto Reduzido de 0,0180 ( $\mathrm{F}=0,312 ; \mathrm{N}=19)$.

Não apresentando o tamanho do individuo arbóreo abatido uma relação com o tamanho de clareira, apontam-se, dessa maneira, as técnicas utilizadas na exploração como as principais responsáveis pelos danos e pelo tamanho da clareira.

Finalmente, a modalidade de exploração utilizada influencia nas características ambientais. Conforme aqui visto, as clareiras geradas a partir da Exploração de Impacto Reduzido estariam fornecendo um ambiente favorável para a sua cicatrização, devido principalmente ao menor tamanho de clareiras. Com base em Campanello (2004) e Campanello et al. (2006), esse menor tamanho de clareira não permitiria a colonização por espécies pioneiras oportunistas, sendo as taquaras dos gêneros Merostachys e Chusquea as de maior destaque na província de Misiones, sendo a primeira a mais agressiva das duas espécies. Elas interceptam a radiação solar, não permitindo a regeneração de espécies arbóreas. O gênero Guadua também é bastante comum na região e igualmente agressivo.

\section{Estimativa da cobertura vegetal}

Os dados obtidos com auxílio de fotografias hemisféricas indicam a tendência de que quanto maior é a altura da leitura da cobertura vegetal, menor é o valor obtido (Figura 3). Isso é justificado pela diluição do número de indivíduos do sub-bosque na medida em que se avança em direção ao dossel da floresta, concordando com os resultados obtidos por Campanello (2004) e Campanello et al. (2004), os quais responsabilizam as taquaras como principais ocupantes do sub-bosque.

Observou-se que entre parcelas do mesmo tratamento existem pequenas diferenças de cobertura, as quais poderiam estar relacionadas a uma composição florística diferenciada entre elas, dada principalmente pela variação de porte que existe entre espécies devido à idade, à heterogeneidade do solo e ao regime hídrico das vertentes. Dessa forma, a dominância de algumas espécies com um porte determinado estaria influenciando o dossel arbóreo das parcelas em estudo.

Quando consideradas as médias por tratamento, mesmo tendo transcorrido 9 anos da ação supressiva, já que a avaliação foi feita em 2007, observa-se que a cobertura ainda é maior nas parcelas testemunhas, e, entre as diferentes modalidades de exploração, o Impacto Reduzido apresenta uma maior cobertura, em ambos os casos para as duas leituras da cobertura vegetal.

As médias dos valores de cobertura resultaram ser estatisticamente não significativas, uma vez que o resultado da ANOVA para as fotografias tiradas a 0,7 e 1,5 metros de altura deram valores de $p$ de 0,1994 e 0,1174 , respectivamente.

A média de cobertura de dossel estimada no presente trabalho é próxima da porcentagem determinada por Johns et al. (1996), os quais encontraram um valor de 91,4\% de cobertura de dossel para uma área sem intervenção.

As porcentagens de perda de dossel estimadas neste trabalho, para cada tratamento de exploração, foram de 15,5\% para Exploração Convencional e de 12,3\% para Exploração de Impacto Reduzido, enquanto que as percentagens obtidas por Johns et al. (1996) foram de 19\% para uma área sem planejamento e de $10 \%$ de perda de dossel para uma área com planejamento.

A figura 4 é ilustrativa da cobertura de dossel na parcela testemunha e da perda dessa cobertura quando feita a exploração nas diferentes modalidades. Cada uma das situações ilustradas foi selecionada com o critério de apresentar um valor de cobertura de dossel mais próximo possível da cobertura média determinada em cada tratamento.

É importante mencionar que as percentagens de cobertura do dossel determinadas para cada tratamento não refletem a cobertura real do dossel, dada exclusivamente pelas árvores estabelecidas que atingem o estrato superior. Esses altos percentuais de cobertura de dossel são resultantes da contribuição também das espécies do sub-bosque, cujas alturas superam dois metros, principalmente em áreas exploradas, onde a ocupação das taquaras é superior quando comparadas com áreas onde a cobertura dada 
pelas copas das árvores no estrato superior é maior. Além disso, deve ser mencionado que as fotografias foram tomadas oito anos depois da aplicação dos tratamentos de exploração.

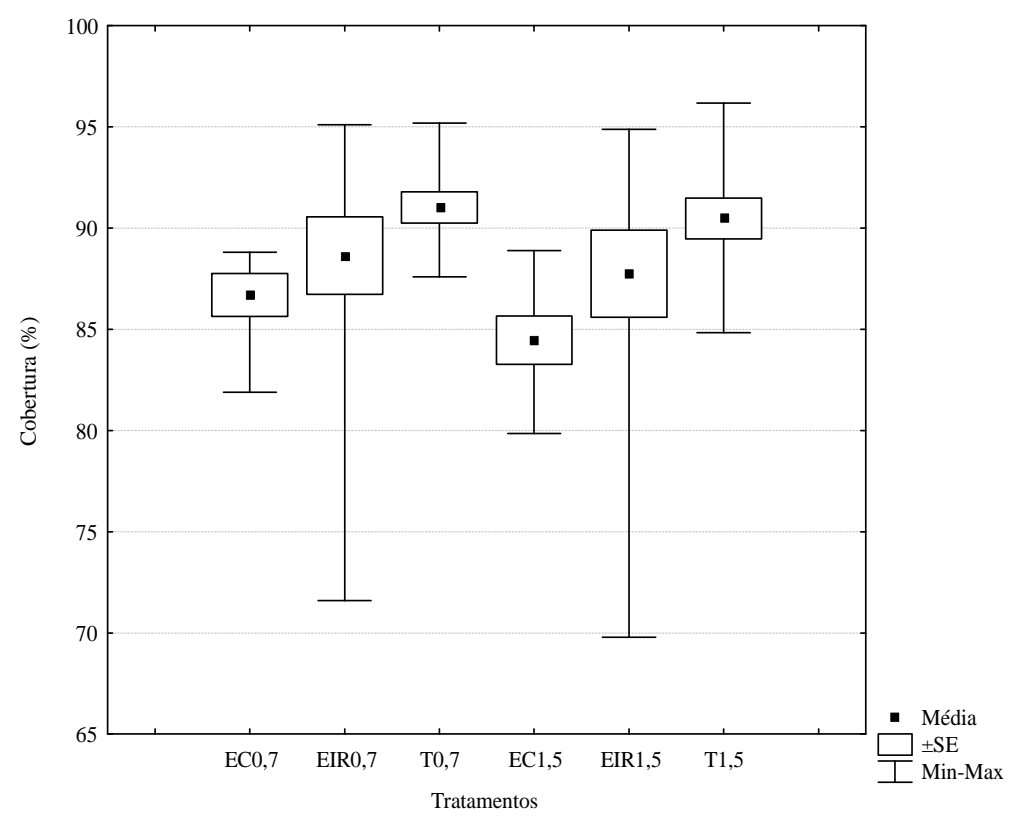

Figura 3. Estimativa de cobertura de dossel por tratamento em duas alturas de observação, 0,7 metros e 1,5 metros, 9 anos após a exploração. EC = Exploração Convencional; EIR = Exploração de Impacto Reduzido e $\mathrm{T}=$ Testemunha.

Figure 3. Estimation of the canopy in each treatment at $0.7 \mathrm{~m}$ and $1.5 \mathrm{~m}$ high, nine years after logging.

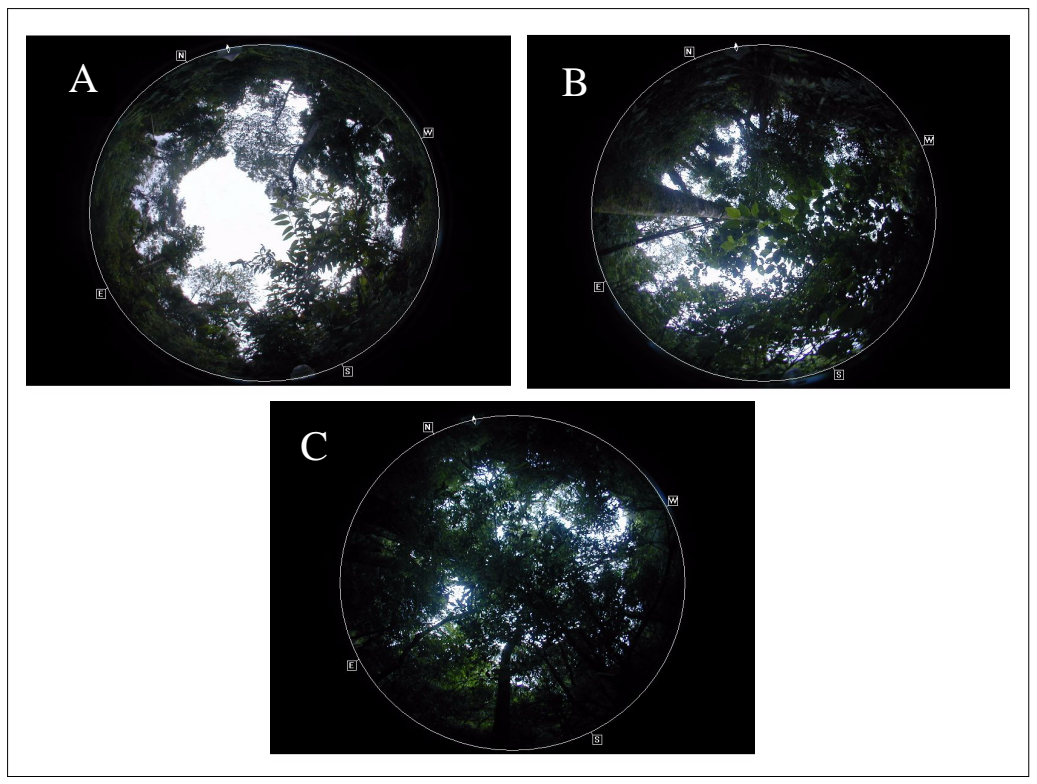

Figura 4. Fotografias hemisféricas da cobertura para cada tratamento a 1,5 metros do solo. $\mathrm{A}=$ Exploração Convencional; B = Exploração de Impacto Reduzido e C $=$ Testemunha.

Figure 4. Hemispherical photographs of the canopy in each treatment at $1.5 \mathrm{~m}$ high. $\mathrm{A}=$ Conventional Logging; B = Reduced Impact Logging and C = Control. 


\section{Danos ocasionados pela exploração aos remanescentes}

Os dois procedimentos de exploração adotados (Impacto Reduzido e Convencional) provocaram danos em 163 indivíduos remanescentes nos 12 ha manejados. O número de indivíduos danificados, quando expressos por unidade de área, foi em média de 13,6 indivíduos por hectare, representando cerca de $4 \%$ do total dos indivíduos estabelecidos na área de ensaio. Em relação ao tamanho dos indivíduos danificados, observou-se que houve uma concentração nas classes diamétricas inferiores, o que permite afirmar que eles estão distribuídos de acordo com a densidade relativa de cada uma das classes diamétricas. Esse comportamento teve a mesma tendência quando analisado para cada tipo de exploração (Figura 5). Esses resultados coincidem com os obtidos por Sist; Ferreira (2007).

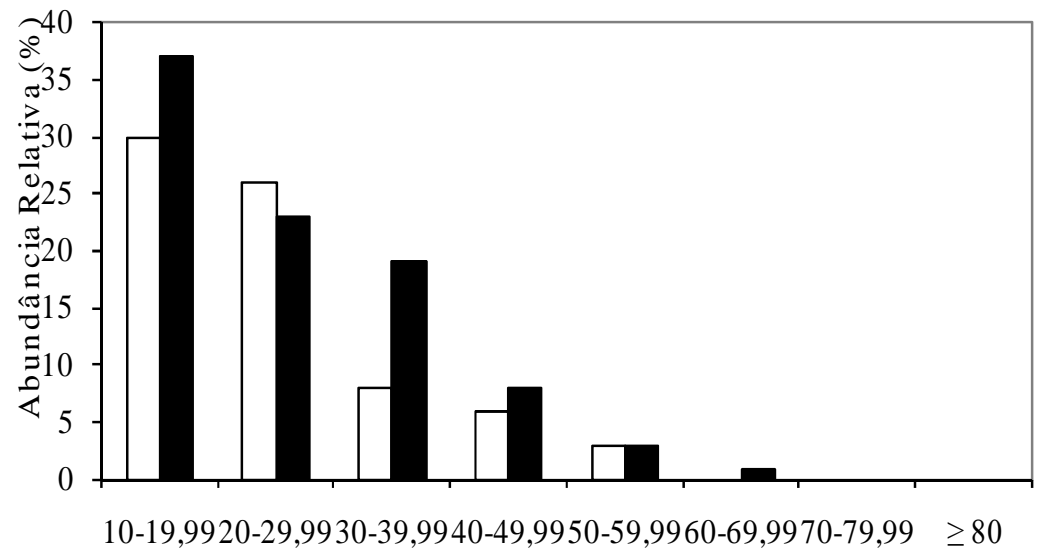

Classes diamétricas $(\mathrm{cm})$

\section{$\square \mathrm{EIR} \quad \mathrm{EC}$}

Figura 5. Distribuição da abundância relativa dos indivíduos danificados por classe diamétrica em cada tratamento de exploração. EIR = Exploração de Impacto Reduzido; EC = Exploração Convencional.

Figure 5. Abundance relative distribution of the number of damaged individuals in each logging system. EIR $=$ Reduced Impact Logging; EC = Conventional Logging.

Comparando as modalidades de exploração, tem-se que, do total dos valores mencionados anteriormente, $44,5 \%$ dos indivíduos foram danificados mediante a aplicação da Exploração de Impacto Reduzido e 55,5\% dos indivíduos foram danificados pela execução da Exploração Convencional. Em valores por hectare, tem-se uma média de 18,2 indivíduos para a Exploração Convencional, o que corresponde a cerca de $6 \%$ dos indivíduos estabelecidos nesse tratamento, e uma média de 10,4 indivíduos para a Exploração de Impacto Reduzido, o que representa 2,9\% dos indivíduos existentes, ou seja, os danos foram reduzidos pela metade quando comparados ao tratamento de maior impacto. Cabe mencionar que a diminuição dos danos com a implementação do Impacto Reduzido estaria sendo influenciada principalmente pela aplicação do abate dirigido, concordando com o mencionado por Johns et al., (1996), Webb (1997) e Pereira et al., (2002), para os quais o abate dirigido resultou ser uma das principais atividades redutoras dos danos aos remanescentes. Os valores de danos registrados por Sist et al. (1998) e Vidal et al. (2002) são superiores aos mencionados aqui, os quais encontraram valores de até $48 \%$ para áreas sob exploração sem planejamento e de $30 \%$ para aquelas planejadas.

Uma das justificativas para essa discrepância poderia estar relacionada às diferenças estruturais das florestas estudadas, especialmente a densidade: florestas com densidade maior apresentariam uma percentagem superior de indivíduos danificados.

Considerando a origem dos danos, o abate destaca-se como a principal atividade que ocasionou um maior número de indivíduos remanescentes danificados para ambos os tipos de exploração. Fazendo a 
comparação entre os tratamentos, observou-se na Exploração Convencional um número maior de indivíduos com esse tipo de dano.

Quando se leva em consideração a parte da árvore que foi danificada, verifica-se que os danos à copa tiveram maior frequência, concordando com afirmativas feitas por Bertault; Sist (1997). Cabe mencionar que os indivíduos com danos na copa apresentaram valores próximos do máximo (100\%). Quando se diferencia por tipo de exploração, nota-se que, na Exploração Convencional, tanto os danos à copa como ao fuste apresentaram um maior número de indivíduos danificados.

Admitindo-se que a principal causa dos danos foi pela queda das árvores abatidas e que a parte da árvore mais afetada foi a copa, pode-se inferir que a primeira é a principal causa da segunda, concordando com Sist et al. (1998) e Bertault; Sist (1997), que mencionam que em cerca de $48 \%$ dos casos de abate ocorrem dano à copa de algum dos remanescentes. Dessa forma, os indivíduos com maiores danos na copa apresentariam um crescimento inferior, já que estariam com a capacidade fotossintética restringida.

Na figura 6, pode ser observada a relação positiva entre a área basal explorada e a área basal danificada em cada parcela explorada $\left(\mathrm{R}^{2}=0,49 ; \mathrm{F}_{(1,10)}=9,752 ; \mathrm{N}=12\right)$. Tendências parecidas foram determinadas pelos autores Bertault; Sist (1997), os quais mencionam uma correlação positiva entre árvores danificadas e a área basal explorada $(\mathrm{r}=0,62)$.

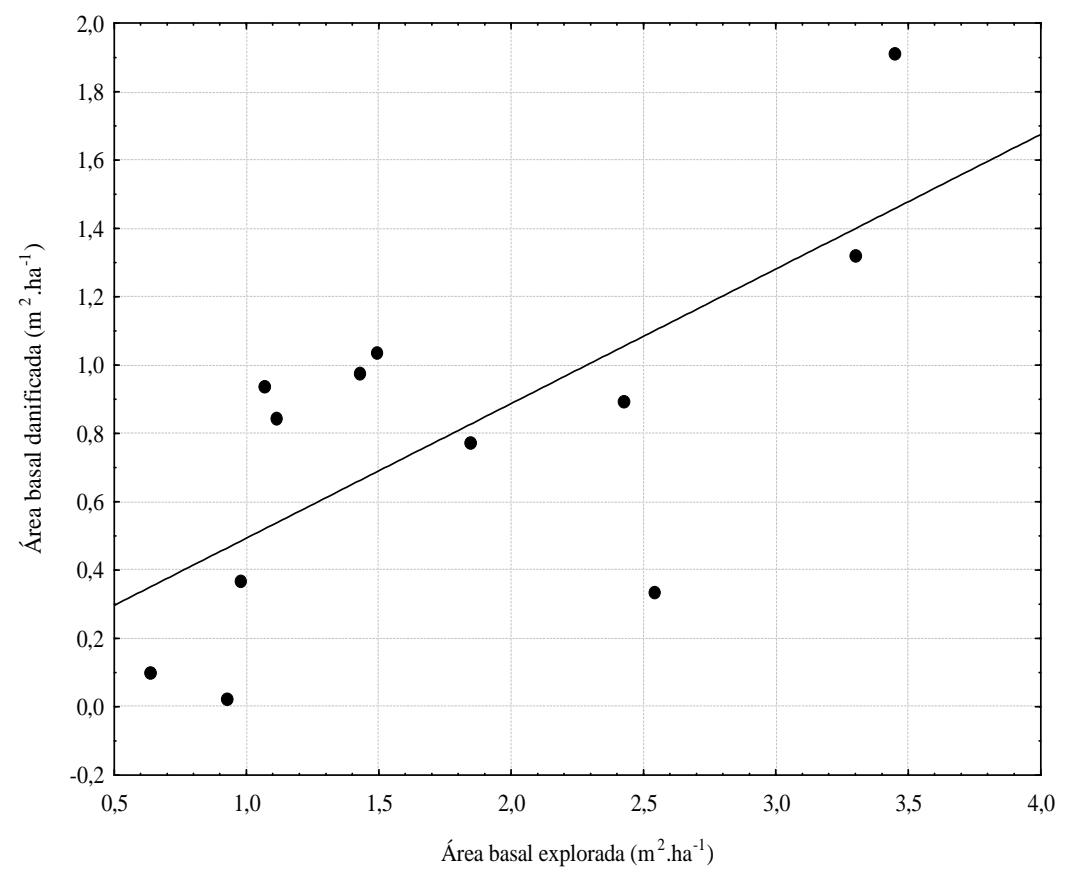

Figura 6. Relação entre a área basal explorada e a área basal danificada em cada parcela submetida a manejo florestal ( $\mathrm{N}=12$ parcelas permanentes exploradas).

Figure 6. Relationship between harvested and damaged basal area in each managed plot $(\mathrm{N}=12$ logged permanent plots).

Quando realizadas as regressões por tratamentos de exploração, observa-se que para Exploração de Impacto Reduzido as variáveis não apresentam uma relação entre elas, diferentemente do observado na Exploração Convencional (Figura 7). Mais uma vez, fica em evidência a influência das técnicas de exploração sobre os remanescentes e a necessidade do planejamento. 


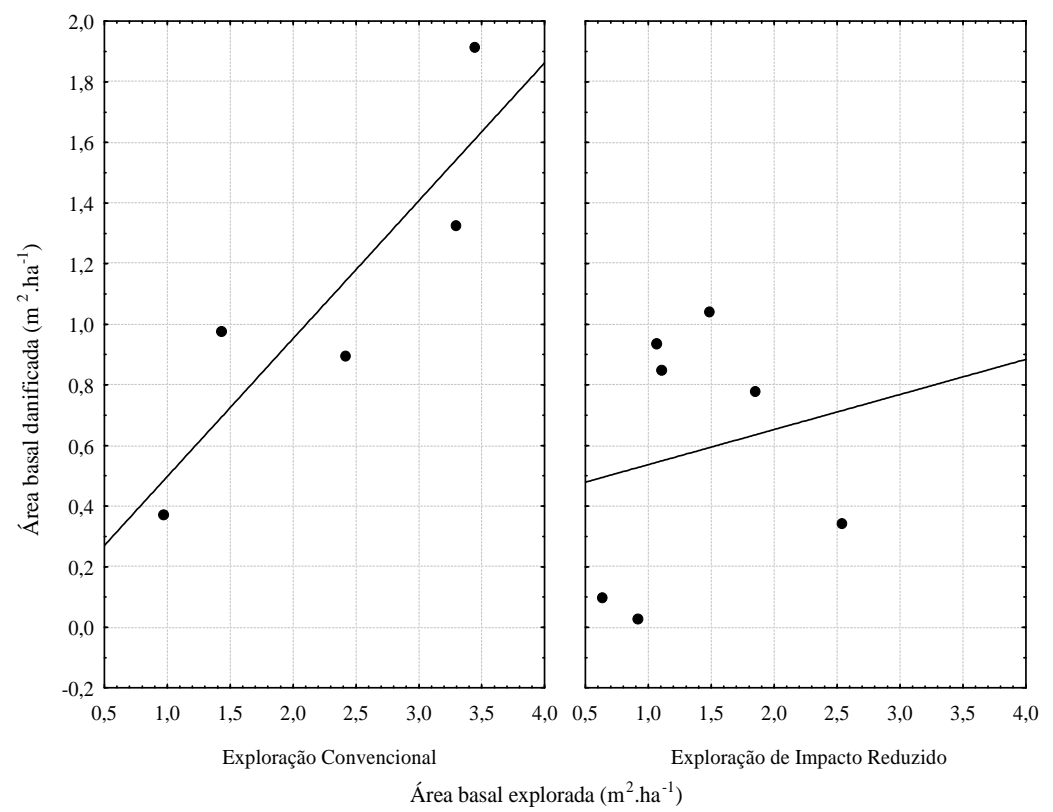

Figura 7. Relação entre a área basal explorada e a área basal danificada em cada tratamento de exploração: Exploração Convencional $\left(\mathrm{R}^{2}=77,2 \% ; \mathrm{F}_{(1,3)}=10,152 ; \mathrm{N}=5\right)$ e Exploração de Impacto Reduzido $\left(\mathrm{R}^{2}=3,2 \% ; \mathrm{F}_{(1,5)}=0,166 ; \mathrm{N}=7\right)$.

Figure 7. Relationship between harvested and damaged basal area in each managed plot, Conventional Logging $\left(\mathrm{R}^{2}=77,2 \% ; \mathrm{F}_{(1,3)}=10,152 ; \mathrm{N}=5\right)$ and Reduced Impact Logging $\left(\mathrm{R}^{2}=3,2 \% ; \mathrm{F}_{(1,5)}=\right.$ $0,166 ; \mathrm{N}=7)$

\section{CONCLUSÕES E RECOMENDAÇÕES}

As clareiras geradas a partir da exploração apresentam características estruturais diferentes em cada modalidade de exploração, sendo de vital importância o planejamento das atividades de colheita florestal.

As percentagens de perda de dossel entre as diferentes modalidades de exploração foram inferiores para a Exploração de Impacto Reduzido. A recuperação da cobertura é considerada como rápida, tendo presente o curto período estudado, sendo os valores de ambos os tratamentos de exploração muito parecidos aos das testemunhas.

A composição florística não foi diferente entre áreas com e sem manejo. O período de estudo (oito anos) poderia ser considerado curto para pretender encontrar diferenças nesse parâmetro.

Dentre as técnicas utilizadas na exploração, a derrubada direcionada demonstrou ser uma das atividades capazes de controlar uma proporção importante dos danos ocasionados aos remanescentes. Houve uma relação significativa e positiva entre a área basal explorada e a área basal danificada para a metodologia de Exploração Convencional.

Tendo em consideração que a exploração das florestas na província de Misiones é legalmente permitida em algumas áreas, a alternativa de Exploração de Impacto Reduzido poderia ser considerada como uma possibilidade menos agressiva quando comparada às técnicas usuais de exploração, não assegurando, necessariamente, a sustentabilidade da floresta. É importante mencionar que essa afirmativa fundamenta-se apenas no componente arbóreo, sem considerar, portanto, outras formas de vida vegetal, igualmente fundamentais para o equilíbrio dos ecossistemas, como herbáceas, epífitas e lianas.

A permissividade da exploração florestal na província de Misiones reproduz um modelo similar ao que já ocorreu na região Sul do Brasil, sob a mesma litologia (ígneas extrusivas), o qual consistiu na substituição da cobertura vegetal original por sistemas agrícolas ou monocultivos de espécies florestais, levando, além da óbvia perda de diversidade tanto vegetal como animal, a sérias consequências em relação a recursos como solo e principalmente água. 


\section{REFERÊNCIAS}

BERTAULT, J. G.; SIST P. An experimental comparison of different harvesting intensities with reducedimpact and conventional logging in East Kalimantan, Indonesia. Forest Ecology and Management. Amsterdam, v. 94, p. 209-218, 1997.

BROKAW, N. The definition of treefall gaps and its effect on measures of forest dynamics. Biotropica, Washington, DC, v. 14, p. 158-160. 1982.

CABRERA, A. L.; WILLINK, A. Biogeografía de América Latina. Washington, DC: OEA, 1980. 117 p. (Monografía, n. 13).

CABRERA, A. L. Territorios Fitogeográficos de la República Argentina. Boletín de la Sociedad Argentina de Botánica, Córdoba, v. 4, n. 1-, p. 1-21, 1951.

CAMPANELLO, P. Diversidad, crecimiento y fisiología de árboles en la selva Misionera: efectos de los cambios en la estructura y funcionamiento del ecosistema producidos por la invasión de lianas y bambúseas. Tesis doctoral. Facultad de Ciencias Exactas y Naturales-Universidad de Buenos Aires. Buenos Aires, Argentina. 2004. 153 p.

CAMPANELLO, P.; MONTTI, L.; GATTI, G.; BRAVO, S.; GOLDSTEIN, G. 2006. Forest structure and functioning in the semideciduous atlantic forest of northern Argentina: effects of native invasive bamboos and lianas on tree regeneration and diversity. In: WORKSHOP ON FOREST FREGMENTATION IN SOUTH AMERICA. San Carlos de Bariloche, Argentina. CD.

EIBL, B.; GONSESKI, D.; BOBADILlA, A.; SILVA, F.; WEBWR, E.; ARTUS, H. Boletín Agrometeorológico, Misiones. Año 7, n. 1, Enero - Diciembre 1995

FERREIRA LIMA, R. A. 2005. Estrutura e regeneração de clareiras em Florestas Pluviais Tropicais. Revista Brasileira de Botânica. v. 28, n. 4, p. 651-670.

GAUTO, O. Análise da Dinâmica e Impactos da Exploração sobre o Estoque Remanescente (por espécie e por grupos de espécies similares) de uma Floresta Estacional Semidecidual em Misiones, Argentina. 133 f. Dissertação (Mestrado em Engenharia Florestal) - Setor de Ciências Agrárias, Universidade Federal do Paraná. Curitiba, 1997.

JACKSON, S.; FREDERICKSEN, T.; MALCOLM, J. Area disturbed and residual stand damage following logging in a Bolivian tropical forest. Forest Ecology and Management. Amsterdam, v. 166, p. 271-283, 2002.

JOHNS, J.; BARRETO, P.; UHL, C. Logging damage during planned and unplanned logging operations in the eastern Amazon. Forest Ecology and Management. Amsterdam, v. 89, p 59-77, 1996.

LAWTON, R. O.; PUTZ, F. E. Natural disturbance and gap-phase regeneration in a wind-exposed tropical cloud forest. Ecology, Tempe, v. 69, n. 3, p.764-777, 1988.

LIGIER, H.; MATTEIO, H.; POLO, J.; ROSSO, J. Província de Misiones. In: Atlas de Suelos de la Republica Argentina. Castelar: INTA-CIRN, 1990. Tomo 2, p 107-154.

MAC DONAGH, P.; GARIBALDI, J.; RIVERO, L.; LAURA SNOOK; TOMA, T. Comparación de daños, mortalidad y reclutamiento em dos sistemas de aprovechamiento forestal en Misiones, Argentina. DÉCIMAS JORNADAS TÉCNICAS FORESTALES Y AMBIENTALES.Eldorado, Misiones. 2005.

MARTINEZ CROVETTO, R. Esquema Fitogeográfico de la Provincia de Misiones. Bomplandia, Corrientes, t.1, n. 3, p.171-223, 1963.

PAHR, N.; FERNÁNDEZ, R.; O'LERY, H.; LUPI, A. Relevamiento edafológico del Área Experimental y Demostrativa "Guaranî" de la Facultad de Ciencias Forestales (UNaM) - Escala 1:50.000. Missiones: Universidad Nacional de Misiones - Facultad de Ciencias Forestales - Instituto Subtropical de Investigaciones Forestales. 1997. 39 p. (Documento Técnico). 
PALAVECINO, J.; MAIOCCO, D. Levantamiento del medio físico del área de investigación forestal Guaraní, Misiones. Eldorado: Universidad Nacional de Misiones. Yvyraretá, Misiones, n. 6, p. 50-63, 1995.

PEREIRA, R.; ZWLEDE, J.; ASNER, G.; KELLER, M. Forest canopy damage and recovery in reduce impact and convencional selective logging in eastern Para, Brazil. Forest Ecology and Management. Amsterdam, v. 168, p. 77-89. 2002.

RUNKLE, J. R. Gap formation in some old-growth forests of the eastern United States. Ecology, Tempe, v. 62, p. 1041-1051, 1981.

SIST, P.; FERREIRA, F. N. Sustainabily of reduced-impact logging in the Eastern Amazon. Forest Ecology and Management. Amsterdam, v. 243, p. 199-209, 2007.

SIST, P.; NGUYEN-THÉ, N. Logging damage and the subsequent dynamics of a dipterocarp forest in East Kalimantan (1990-1996). Forest Ecology and Management, Amsterdam, v. 165, p. 85-103, 2002.

SIST, P.; NOLAN, T.; BERTAULT, J.; DYKSTRA, D. Harvesting intensity versus sustainability in Indonesia. Forest Ecology and Management, Amsterdam, v. 108, p. 251-260, 1998.

TRESSENS, S.; REVILLA, V. Riqueza florística de un bosque nativo de 5000 Hectáreas conducido racionalmente en la provincia de Misiones. II Congreso Argentino y Latinoamericano. Bosque nativo y protección ambiental. AfoA. Del 13 al 15 de Agosto. Posadas, Misiones. 1997.

VELOSO, H. P.; GÓES-FILHO, L. Fitogeografia Brasileira. Classificação fisionômico-ecológica da vegetação neotropical. Boletim Técnico Projeto RADAMBRASIL. Série vegetação, Salvador, 85 p, 1982.

VIDAL, E.; VIANA, M.; FERREIRA BATISTA, J. Crescimento de floresta tropical três anos após colheita de madeira com e sem manejo florestal na Amazônia oriental. Scientia Forestalis, Piracicaba, n. 61, p. 133-143, 2002.

WEBB, E. Canopy removal and residual stand damage during controlled selective logging in lowland swamp forest of northeast Costa Rica. Forest Ecology and Management, Amsterdam, v. 95, p 117-129, 1997. 